\title{
Autoimmune encephalitis: the new hit at neurological parade
}

\author{
Encefalite autoimune: a nova sensação na parada neurológica
}

Angelina Maria Martins Lino

\author{
Neurologist, MD, PhD; Division of \\ Neurology, Hospital das Clínicas, \\ São Paulo University School of \\ Medicine, Division of Internal \\ Medicine, Hospital Universitário, \\ São Paulo University, São Paulo SP, \\ Brazil. \\ Correspondence: \\ Angelina Maria Martins Lino \\ Divisão de Clínica Neurológica, \\ Instituto Central \\ Avenida Doutor Enéas de Carvalho \\ Aguiar $255 / 5^{\circ}$ andar \\ 05403-900 São Paulo SP - Brasil \\ E-mail:angelina@usp.br \\ Conflict of interest \\ There is no conflict of interest to \\ declare. \\ Received 23 August 2012 \\ Accepted 30 August 2012
}

$\mathrm{R}$ ecently, we have witnessed the fast growth of knowledge in neurology, particularly within neuroimmunology. Also, old neurological diseases have been revisited and renamed with the emerging class of anti-neural antibodies (AN-Ab). At the same time, neurologists have become aware of diagnosing immune-mediated diseases and understanding their immunological mechanisms. Different lines of research have uncovered that peripheral and central nervous tissues are not immune-privileged sites, which used to be thought due to their biological barriers.

Independently of terminology ${ }^{1,2}$, we should bear in mind that autoimmune encephalitis (AIE) is a generic term that encompasses more than paraneoplastic and non-paraneoplastic diseases or conditions linked to AN-Ab. For instance, Machado et al. ${ }^{3}$, in this issue of Arquivos de Neuropsiquiatria, and other classical revisions ${ }^{3-8}$ have shown that not only the neurological manifestations are shared by other more common neurological diseases, but also one specific $\mathrm{AN}-\mathrm{Ab}$ is associated with clinical heterogeneity and distinct neurological prognosis.

Unquestionably, the AN-Ab discovery has contributed for our understanding of AIE pathogenic process ${ }^{9,10}$, and can point to a more specific treatment in near future. However, we should deal with some issues. For example, the infectious diseases are highly prevalent, these new $\mathrm{AN}-\mathrm{Ab}$ laboratorial tests are not easily and routinely available in public health system (even at university centers), the costs of them privately held are very expensive ( $\$ 1,000-2,000 / \mathrm{Ab} /$ sample), their results take at least 20 days to be released, even privately held or kindly given by doctor Dalmau (problematic when we are face to a case of acute onset), the AN-Ab negative results do not exclude AIE diagnosis, and some medicines used abroad have restrictive practices, such as monoclonal antibodies in Brazil.

Considering literature data, the previous paragraph, and our own service experience, it can be noted that AIE could be diagnosed and treated without any AN-Ab tests. In a more chronic presentation (more than six months ${ }^{7}$ ) at first neurological evaluation, all steps presented by Machado et al. should be followed, and the immunotherapy should be started as soon as the more common diseases have been ruled out.

The major concern is represented by acute/sub-acute onset (less than six weeks ${ }^{2}$ ) of altered mental status, in which the main step is promptly to think, investigate, and treat, even empirically, an infectious disease (specially treatable encephalitogenic virus). However, it must take into account the computed tomography (or rather, magnetic resonance image) and cerebrospinal fluid characteristics. Infection is the only condition that could be seriously worsened if the immunotherapy (corticosteroids, plasma exchange, or other immunosuppressive drug) was started. Normally, metabolic, deficiency, and toxic disorders are easily and readily ruled out (it is advisable include serum ammonia quantification where alcoholism is frequent or there is personal history of alcohol abuse). Called by the authors as autoimmune meningoencephalitis, this is a large group of inflammatory noninfectious encephalopathies that could present major difficulties when there is no systemic involvement (skin, lung, kidney, joints, or other organ) to guide the diagnosis. In practice, this group share not only inflammatory mechanisms, but also the same unspecific immunotherapy used in AIE. It is agreed that cancer investigation has to be carried out in all patients, and there is no difference in their treatment while it is negative. Nevertheless, we should bear in mind that some confirmed neoplastic patients could also need 
immunotherapy for their neurological disease, which had not improved with oncologic treatment.

As shown by Machado et al. in this issue, there is almost a consensus in literature on the initial treatment for AIE. What should we do after that? At evidence-based medicine era, the long-term treatment is still based on experience. Based on our long service experience with typical and atypical, paraneoplastic and non-paraneoplastic, peripheral and central nervous system autoimmune diseases, it is suggested that monthly methylprednisolone should be maintained for at least a six-month period (cyclophosphamide should not be postponed depending on disease severity). Following these pulses, the patients should receive long-term oral immunosuppressive treatment, by similarity to protocols for rheumatologic diseases. Depending on clinical evolution in particular cases, other therapeutic options could be added.

Finally, this expanding class of AN-Ab with its pathogenic roles and correlation with clinical and functional prognosis are of undeniable importance. The greatest challenge is to apply this new knowledge ${ }^{1-9}$ to reduce morbidity and mortality in AIE patients. The insight for AIE diagnosis comes from methodical clinical reasoning, which includes an early and complete clinical history allied to the critical analysis of the tests' results and images, and closely monitoring of clinical events. Considering the need for fast and complete laboratory and imaging research, these patients should be referred to more equipped and experienced centers.

\section{References}

1. Pruitt AA. Immune-mediated encephalopathies with an emphasis on paraneoplastic encephalopathies. Semin Neurol 2011;31:158-168.

2. Flanagan EP, Caselli RJ. Autoimmune encephalopathy. Semin Neurol 2011;31:144-157.

3. Machado S, Pinto AM, Irani SR. What you should know about autoimmune encephalitis. Arq Neuropsiquiatr 2012;70:817-822.

4. Tan KM, Lennon VA, Klein CJ, Boeve BF, Pittock SJ. Clinical spectrum of voltage-gated potassium channel autoimmunity. Neurology 2008;70:1883-1890

5. Rosenfeld MR, Dalmau J. Update on paraneoplastic and autoimmune disorders of the central nervous system. Semin Neurol 2010;30:320-331.
6. Irani SR, Vincent A. Autoimmune encephalitis - new awareness, challenging questions. Discov Med 2011;11:449-458.

7. Vincent A. Autoimmune channelopathies: well-established and emerging immunotherapy-responsive diseases of peripheral and central nervous system. J Clin Immunol 2010;30(Suppl 1):S97-S102.

8. Vincent A, Bien CG, Irani SR, Waters P. Autoantibodies associated with diseases of the CNS: new developments and future challenges. Lancet Neurol 2011;10:759-772.

9. Lee R, Buckley C, Irani SR, VincentA. Republished review: autoantibody testing in encephalopathies. Postgrad Med J 2012;88:280-292.

10. Bien CG, Vincent A, Barnett $\mathrm{MH}$, et al. Immunopathology of autoantibody associated encephalitides: clues for pathogenesis. Brain 2012;135:1622-1638. 